\title{
Scalable Ultrasmall Three-Dimensional Nanowire Transistor Probes for Intracellular Recording
}

\section{Citation}

Zhao, Yunlong, Siheng Sean You, Anqi Zhang, Jae-Hyun Lee, Jinlin Huang, and Charles M. Lieber. 2019. Scalable Ultrasmall Three-dimensional Nanowire Transistor Probes for Intracellular Recording. Nature Nanotechnology 14, no. 8: 783-90.

\section{Permanent link}

http://nrs.harvard.edu/urn-3:HUL.InstRepos:42241193

\section{Terms of Use}

This article was downloaded from Harvard University's DASH repository, and is made available under the terms and conditions applicable to Other Posted Material, as set forth at http:// nrs.harvard.edu/urn-3:HUL.InstRepos:dash.current.terms-of-use\#LAA

\section{Share Your Story}

The Harvard community has made this article openly available.

Please share how this access benefits you. Submit a story.

Accessibility 
1 Title: Scalable ultrasmall three-dimensional nanowire transistor probes for

2 intracellular recording

4 Authors: Yunlong Zhao ${ }^{1,2 \dagger}$, Siheng Sean You ${ }^{1 \dagger}$, Anqi Zhang ${ }^{1 \dagger}$, Jae-Hyun Lee ${ }^{1,3}$, Jinlin Huang ${ }^{1}$ 5 and Charles M. Lieber ${ }^{1,4,5 *}$

6

7 Affiliations:

$8{ }^{1}$ Department of Chemistry and Chemical Biology, Harvard University, Cambridge, 9 Massachusetts 02138, USA

$10 \quad{ }^{2}$ Advanced Technology Institute, University of Surrey, Guildford, Surrey GU2 7XH, UK

$11{ }^{3}$ Center for Nanomedicine, Institute for Basic Science (IBS), Yonsei-IBS Institute, Yonsei

12 University, Seoul 03722, Republic of Korea

$13{ }^{4}$ Center for Brain Science, Harvard University, Cambridge, Massachusetts 02138, USA

$14{ }^{5}$ John A. Paulson School of Engineering and Applied Sciences, Harvard University, Cambridge,

15 Massachusetts 02138, USA

$16 \uparrow$ These authors contributed equally to this work.

$17 *$ *e-mail: cml@cmliris.harvard.edu 


\section{Abstract}

19 New tools for intracellular electrophysiology that push the limits of spatiotemporal resolution

20 while reducing invasiveness could provide a deeper understanding of electrogenic cells and their

21 networks in tissues and push progress towards human-machine interfaces. While significant

22 advances have been made in developing nanodevices for intracellular probes, current approaches

23 exhibit a tradeoff between device scalability and recording amplitude. We address this challenge

24 by combining deterministic shape-controlled nanowire transfer with spatially-defined

25 semiconductor-to-metal transformation to realize scalable nanowire field-effect transistor probe

26 arrays with controllable tip geometry and sensor size, which enable recording of up to $100 \mathrm{mV}$

27 intracellular action potentials from primary neurons. Systematic studies on neurons and

28 cardiomyocytes show that controlling device curvature and sensor size is critical for achieving

29 high amplitude intracellular recordings. In addition, this device design allows for multiplexed

30 recording from single cells and cell networks and could enable future investigations of dynamics

31 in the brain and other tissues.

32 
Developing new tools that enable reproducible high spatial-temporal resolution recording

35 of intracellular potential, while maintaining the capability for device scalability, are key goals for 36 advancing electrophysiology studies of electrogenic cells and cell networks ${ }^{1-4}$. Patch-clamp

37 electrodes have been the "gold standard" for cell electrophysiology for decades, where it has

38 been shown that accurate recording of the intracellular potential requires a high resistance seal 39 against the cell membrane and low resistance between the recording element and the cell 40 interior ${ }^{5,6}$. Recent studies have focused on several solid-state nanodevice architectures, including 41 nanowire-based structures for optical neuronal stimulation ${ }^{7,8}$, scalable on-chip micro/nano42 structured electrode arrays ${ }^{9,10}$ for attenuated intracellular recording via electroporation ${ }^{11,12}$ and/or 43 optoporation ${ }^{13,14}$, and three-dimensional nanowire field effect transistor probes for intracellular 44 recording of single cells ${ }^{15,16}$. Nanowire probes have recorded cardiac intracellular action 45 potentials with amplitudes comparable to those recorded with patch-clamp micropipettes ${ }^{15,16}$, but 46 have relied on 1-by-1 fabrication that has been difficult to scale-up. For solid-state

47 nanoprobes to achieve comparable recording signal-to-noise and amplitude to patch clamp, the 48 nanodevice must achieve direct contact of the recording element with the intracellular solution 49 without significantly disturbing the cell membrane ${ }^{2,} 9$. Fulfilling these criteria requires 50 understanding of the size, geometry, mechanical, and biochemical factors present at the cell 51 membrane/nanodevice interface. Recent work suggests that nanoscale size and geometry play a 52 key role in the interaction between the nanostructure and the cell membrane ${ }^{17,18}$. For example, 53 nanoscale membrane curvature elevates the local concentration of endocytosis related 54 proteins $^{17,18}$, influences the conformation and activity of transmembrane proteins ${ }^{19}$, and is 55 hypothesized to recruit a sequence of proteins leading to membrane fission ${ }^{20}$. Building on these 
studies, we hypothesize that inducing appropriate nanoscale curvature on the cell membrane via

57 rational device design will facilitate probe internalization and enable intracellular recording.

Here, we investigate how size and geometry of nanoprobes influence intracellular

59 recording by fabricating scalable three-dimensional U-shaped nanowire field effect transistor (U-

60

61

62

63

64

65

66

67

68

69

70

71

72

NWFET) arrays in which both the radii of curvature (ROC) and active sensor sizes are controlled

(Fig. 1a). To investigate how these design factors affect electrophysiological recording, arrays of U-NWFET probes fabricated from $15 \mathrm{~nm}$ diameter p-type Si nanowires with ROC from $0.75-2$ $\mu \mathrm{m}$ and active channel lengths from $50-2000 \mathrm{~nm}$ were used to probe cultured primary neurons and human cardiomyocytes. Schematically, we ask whether probes with the smallest ROC and sensor size (Fig. 1b(i)) can facilitate recording full amplitude intracellular action potentials and subthreshold features, whereas increases in the ROC and detector sizes (Fig. 1b(ii)) would lead to recording smaller amplitude intracellular-like or extracellular action potential peaks.

\section{U-shaped nanowire probe fabrication and characterization}

Our strategy for producing reproducible arrays of U-NWFET probes with controlled ROC and active FET channel lengths or detector sizes involves two key techniques (see Methods for details). First, large-scale, shape-controlled deterministic assembly ${ }^{21}$ is used to produce Ushaped nanowire arrays from $15 \mathrm{~nm}$ diameter Si nanowires with controllable ROC on top of $\mathrm{Si}_{3} \mathrm{~N}_{4}$ patterns (Fig. 2a(i); Supplementary Fig. 1a-e). Metal contacts are then deposited and passivated by an upper $\mathrm{Si}_{3} \mathrm{~N}_{4}$ layer (Supplementary Fig. 1f). Second, we exploit spatially-defined solid-state transformation ${ }^{22}$ to convert $\mathrm{Si}$ nanowire segments underneath and adjacent to $\mathrm{Ni}$ diffusion layer to metallic NiSi, thereby producing controlled length of FET sensing elements at the tips of the U-shaped nanowire probes (Fig. 2a(ii); Supplementary Fig. 1g). Last, etching of the sacrificial layer allows the probes to bend upward due to interfacial strain in the metal 
79 interconnects (Fig. 2a(iii)) ${ }^{15}$, yielding probe arrays with up to 4 addressable U-NWFETs per 80 bend-up probe arm (Supplementary Fig. 1h).

Optical microscopy and scanning electron microscopy (SEM) were used to characterize

82 key steps in the U-NWFET probe fabrication flow. Optical microscopy images of the patterned

83 bottom passivation layer and U-shaped trenches that set the ROC during nanowire assembly

84 (Supplementary Fig. 2a,b) as well as three probes with U-shaped nanowires, metal contacts and top passivation layer (Fig. 2b) are indicative of the deterministic parallel assembly of U-shaped nanowire probes with defined ROC. Composition-sensitive SEM images of U-NWFETs 87 following annealing of the patterned $\mathrm{Ni}$ further highlight the control of channel lengths from ca. $50 \mathrm{~nm}$ (Fig. 2c) to 500 and $2000 \mathrm{~nm}$ (Supplementary Fig. 2c,d). Measured channel lengths and 89 ROCs were found to be consistent with those designed (Supplementary Fig. 3). Etching of the Ni release layer produces arrays of probes, including single U-NWFET probes (Fig. 2d) and 91 multiple U-NWFET devices on a single probe arm (Supplementary Fig. 2e) where the active U92 NWFET sensor elements are oriented upwards away from the substrate.

Electrical transport studies in air and aqueous solution were carried out to characterize 94 the sensor properties. Current versus drain-source voltage $\left(I-V_{d s}\right)$ measurements on devices 95 with channel lengths of ca. 50, 500, and $2000 \mathrm{~nm}$ (Fig. 2e-g; $\mathrm{N}=10$, each channel length) in the 96 dry state yield average conductances of $3.3 \pm 0.6,0.7 \pm 0.2$ and $0.3 \pm 0.1 \mu \mathrm{S}$, respectively. In 97 addition, conductance versus water-gate voltage $\left(\mathrm{V}_{\mathrm{g}}\right)$ measurements in aqueous solution (Fig. 2h98 j) yield average transconductances of $5.4 \pm 1.3,2.3 \pm 0.7$ and $0.9 \pm 0.3 \mu \mathrm{S} / \mathrm{V}$ for 50,500 and $992000 \mathrm{~nm}$ channel lengths, respectively. The probe transconductance versus ROC $(0.75-2.0 \mu \mathrm{m})$ 100 for devices with 50, 500 or 2000 nm FET channels (Supplementary Fig. 4 and Supplementary 101 Table 1) show that transconductance does not significantly vary as a function of ROC in our 
102 designed strain range (Supplementary Table 2). The conductance and transconductance results

103 for the U-NWFETs are roughly consistent with the expected inverse relationship to the channel

104 length. Last, the transconductance and measured noise values yield an estimate for signal 105 detection sensitivity (3 standard deviations) of $0.90 \pm 0.60,1.2 \pm 0.9$ and $1.9 \pm 0.9 \mathrm{mV}$ for 50 , 106500 and $2000 \mathrm{~nm}$ channel lengths, respectively, which should allow detection of typical 1-10 mV 107 subthreshold activities of neurons ${ }^{2}$.

\section{Near full amplitude intracellular recordings}

With these characterization results, we first asked whether ultrasmall U-NWFET probes

110 could record full amplitude intracellular action potentials from primary neurons. First, a single

111 U-NWFET probe with ca. $50 \mathrm{~nm}$ FET length and $0.75 \mu \mathrm{m}$ ROC was used to sequentially

112 measure six independent dorsal root ganglion (DRG) neurons (Methods, Supplementary Fig. 5),

113 where the probe was not remodified with lipid between the sequential measurements

114 (Supplementary Fig. 6). In each trace, we observe a drop in the baseline potential upon initial 115 cell contact (Fig. 3a). Subsequently, either sparse peaks (Cells 1,3,6) or periodic peaks (Cells $1162,4,5)$ are observed with amplitude of $60-100 \mathrm{mV}$ and signal-to-noise ratios of $115 \pm 29$. For 117 each cell, the recorded potentials have consistent shape and duration, and characteristic single 118 peaks recorded from the 6 cells are shown in Fig. 3b. An additional set of data recorded from two 119 DRG neurons without spontaneous firing properties showed a voltage drop (Supplementary Fig. 120 7a), or one single peak followed by a voltage drop (Supplementary Fig. 7b) during device 121 penetration. Following the initial recording, we observed a gradual decrease in the peak 122 amplitude as well as a positive shift in the baseline potential (Fig. 3c, $d$ and Supplementary Fig. 123 7). 

patterns and signal-to-noise ratio of the peaks are similar to our patch-clamp recordings of 126 similarly cultured DRG neurons (Supplementary Fig. 8) and are consistent with the reported 127 heterogeneity of spontaneously firing DRG action potential waveforms and spike patterns ${ }^{23}$. 128 These data thus indicate that the ultrasmall U-NWFETs with biomimetic phospholipid 129 modification can obtain high resistance membrane seals, achieve direct access to the cell interior, 130 and yield faithful recording of the intracellular potential. Notably, the data recorded from some 131 DRG neurons exhibit characteristics consistent with mechanosensitive properties ${ }^{24}$, including an 132 increase in action potential firing rate (Fig. 3c,d) and firing of a single action potential 133 (Supplementary Fig.7b) during formation of the device/cell junction. A limitation of the 134 recording is, however, the shift in baseline and decrease in recorded action potential amplitude at 135 later times (e.g., Fig. 3c). We suggest that these changes are due to either an elastic response 136 from the cytoskeleton, which gradually pushes the probe out of the cell as suggested in other 137 intracellular chemical delivery experiments ${ }^{25}$, or mechanical instability of the measurement 138 setup.

Given the high signal-to-noise for our measurements, we asked whether it was possible to observe subthreshold activity. Notably, close examination of a representative trace from a cell 141 with irregular firing pattern (Fig. 3d) shows subthreshold features, including a single ca. $5 \mathrm{mV}$ 142 peak (Fig. 3e(i)) and a series of three small peaks (Fig. 3e(iii)) immediately prior to the initiation 143 of an action potential, as well as a ca. $3 \mathrm{mV}$ peak not associated with an action potential spike 144 (Fig. 3e(ii)); we recorded similar results with patch-clamp (blue triangles, Supplementary Fig. 8). 145 Previous multi-patch-clamp studies have reported comparable subthreshold signals and attributed 146 them to excitatory postsynaptic potentials in which a presynaptic cell triggers the firing of the 
147 postsynaptic cel1 $1^{2,26}$. This suggests that our U-NWFET devices can measure biologically relevant 148 subthreshold signals and could be used for future studies of neural connections and synaptic 149 activity.

After achieving neuronal intracellular recording, we asked whether the U-NWFET probes could be generalized to other electrogenic cells. To answer this question, we cultured human 152 induced pluripotent stem cell-derived cardiomyocytes (HiPSC-CMs) (Methods). Contact of a 153 HiPSC-CM and a U-NWFET probe with ca. $50 \mathrm{~nm}$ FET length and $0.75 \mu \mathrm{m}$ ROC 154 (Supplementary Fig. 9a,b) initially yielded a ca. $25 \mathrm{mV}$ drop in the baseline, followed by 155 periodic ca. $50 \mathrm{mV}$ positive waveforms with a sharp rising phase $(<50 \mathrm{~ms})$, slow falling phase (ca. $400 \mathrm{~ms}$ ) and frequency of $1.25 \pm 0.04 \mathrm{~Hz}$. The second measurement of the same cell resulted 157 again in a drop in the baseline potential and initial ca. $50 \mathrm{mV}$ positive waveforms with 158 frequencies $1.23 \pm 0.02 \mathrm{~Hz}$ (Supplementary Fig. 9c,d). Notably, the waveform frequency, 159 amplitude and shapes during the first and the second entry remain similar and are consistent with 160 reported cardiac action potentials ${ }^{27}$, suggesting that the U-NWFET probe is also able to record 161 the intracellular potential of cardiac cells, and that the internalization process is minimally 162 invasive.

\section{Effect of nanowire geometry and sensor size on intracellular recording}

We first investigated geometry effects by fabricating probe arrays with ROCs ranging 165 from $0.75 \mu \mathrm{m}$ to $2.0 \mu \mathrm{m}$ (Fig. 4a, Supplementary Fig. 10) with fixed ca. $50 \mathrm{~nm}$ sensor sizes, 166 carrying out ca. 30 measurements for each ROC from both DRG neurons and HiPSC-CMs. For 167 DRG neurons, representative intracellular/intracellular-like recordings were obtained by probes 168 with $1 \mu \mathrm{m}$ and $1.5 \mu \mathrm{m}$ ROC (Fig. 4b, c), showing maximum action potential amplitudes of ca. 35 $169 \mathrm{mV}$ and $12 \mathrm{mV}$ respectively. The distribution of maximum recording amplitudes from both cell 
170 types (Fig. 4d,e) shows the average values for DRG/HiPSC-CM cells and number of successful 171 recordings (out of ca. 30 measurements) of $34 \pm 30(\mathrm{~N}=24) / 34 \pm 14(\mathrm{~N}=31), 19 \pm 15(\mathrm{~N}=25) / 31 \pm 9$

$172(\mathrm{~N}=6)$ and $16 \pm 7(\mathrm{~N}=2) / 21 \pm 9(\mathrm{~N}=7) \mathrm{mV}$ for $0.75,1.0$ and $1.5 \mu \mathrm{m}$ ROC, respectively.

173 Interestingly, the $2.0 \mu \mathrm{m}$ ROC probes did not yield successful recordings on both cell types,

174 indicating that increasing ROC correlates with lower recorded maximum amplitudes.

175 Measurement of device transconductance (Supplementary Fig. 11) and SEM images

176 (Supplementary Fig. 12) indicate that device characteristics do not change following

177 measurement.

Second, we studied how sensor size affects recording by fabricating U-NWFETs with 179 channel lengths of $500 \mathrm{~nm}$ and $0.75 \mu \mathrm{m}$ ROC (Supplementary Fig. 10). Measurements made on 180 both DRG neurons and HiPSC-CMs showed $8 \pm 8(\mathrm{~N}=7)$ and $23 \pm 13 \mathrm{mV}(\mathrm{N}=10)$ maximum 181 intracellular action potential amplitudes, respectively (Fig. 4f). Furthermore, U-NWFETs with 182 channel lengths of ca. $2000 \mathrm{~nm}$ and ROC of either $1.0 \mu \mathrm{m}$ or $1.5 \mu \mathrm{m}$ yielded maximum 183 amplitudes of $21 \pm 12 \mathrm{mV}(\mathrm{N}=5$, blue circles $)$ and $8.0 \mathrm{mV}(\mathrm{N}=1$, yellow circle), respectively

184 (Fig. 4g). Some of the $1.5 \mu \mathrm{m}$ ROC, $2000 \mathrm{~nm}$ channel U-NWFETs recorded negative spikes with 185 maximum amplitudes of $4 \pm 1 \mathrm{mV}(\mathrm{N}=4$, red triangles), while no successful recordings were achieved on DRG cells with $2000 \mathrm{~nm}$ channel probes. A representative trace showing negative 187 spikes from a HiPSC-CM (Fig. 4h) highlights their periodic negative short $<3$ ms duration that contrast a representative intracellular recording ${ }^{27}$ obtained from a HiPSC-CM using $0.75 \mu \mathrm{m}$ 189 ROC, $50 \mathrm{~nm}$ U-NWFET (Fig. 4i). The above data show that reducing the ROC and FET channel 190 length has a statistically significant correlation with the increases in both the maximum measured 191 action potential amplitudes and number of successful recordings (Supplementary Table 3). At the 192 most extreme limit, devices with $2 \mu \mathrm{m}$ ROC no longer record action potentials, although devices 
193 with $1.5 \mu \mathrm{m}$ ROC and $2000 \mathrm{~nm}$ channel lengths recorded waveforms characteristic of 194 extracellular signals ${ }^{27,28}$. We hypothesize that the observed increase in recording amplitude with 195 decreasing ROC and channel length is not solely from physical interactions between the device 196 and the cell but also a consequence of reported results demonstrating that nanoscale curvature 197 can induce activation of endocytosis and related biological pathways. ${ }^{17-20,29}$ Our studies indicate 198 that using nanoscale topography to enhance device uptake is critical for developing tools that 199 faithfully capture intracellular action potential features.

200 We further examined the relationship between U-NWFET device ROC and channel 201 length and the intracellular recording duration. For both DRG neurons and HiPSC-CMs, there 202 was no significant difference ( $\mathrm{p}$-value $>0.05$ ) in recording duration with ROC (Supplementary 203 Fig. 13, Supplementary Table 4). Additionally, we observed that increasing channel lengths 204 showed no difference in recording duration in the DRG cells ( $p$-value $>0.05$ ), while for the 205 HiPSC-CMs channel length increasing from $50 \mathrm{~nm}$ to $500 \mathrm{~nm}$ for the $0.75 \mu \mathrm{m}$ ROC and from 50 $206 \mathrm{~nm}$ to $2000 \mathrm{~nm}$ for the $1.0 \mu \mathrm{m}$ ROC U-NWFET resulted in a statistically significant (p207 values $=0.007,0.027$ respectively) increase in recording duration (Supplementary Fig. 13, 208 Supplementary Table 4). The lack of correlation between ROC and recording distribution 209 suggests loss of intracellular access is related to curvature independent factors. We attribute the 210 observed increased recording duration for longer channel lengths for HiPSC-CMs to the higher 211 probability of maintaining a partially internalized configuration during cell contraction-induced 212 instabilities. Recordings obtained on DRG neurons have shorter duration than HiPSC-CMs, 213 possibly reflecting reported differences in cell membrane mechanical properties as neuron 214 membranes are generally less fluidic than those of cardiac cells ${ }^{30}$. 
Finally, we ask whether deterministic fabrication with size and geometry control could enable multisite intracellular recording within a single cell using two U-NWFETs on one probe 217 arm, recording from cell networks using independent U-NWFET probes, and/or simultaneous 218 measurement of intracellular/extracellular action potentials from a single cell by two U-NWFETs 219 with different ROC and channel lengths (Fig. 5a). First, a single DRG neuron soma was brought 220 into contact with a pair of U-NWFETs (each with ca. $50 \mathrm{~nm}$ channel and $0.75 \mu \mathrm{m}$ ROC) 221 separated by $2 \mu \mathrm{m}$ on one probe arm. The simultaneously recorded intracellular action potential 222 amplitudes (Fig. 5b(i)) exhibited values of 46 and $28 \mathrm{mV}$ from the two U-NWFETs. The 223 derivative of two action potentials signals (Fig.5b(ii)) and overlay of the two traces scaled to the 224 same peak amplitudes (Supplementary Fig. 14a,b) shows that the peaks coincide with each other, 225 indicating there is no discernible delay or waveform difference observed in the soma between the 226 two channels.

227 Second, a layer of cultured HiPSC-CMs was brought into contact with paired U228 NWFETs on the same probe arm (2 $\mu \mathrm{m}$ separation) and a third single U-NWFET probe 229 separated by $350 \mu \mathrm{m}$ from the paired probe (all three U-NWFETs with ca. $50 \mathrm{~nm}$ channel and $230 \quad 0.75 \mu \mathrm{m}$ ROC). The paired probe recorded the intracellular action potential within one cell with 231 action potential amplitudes $54 \mathrm{mV} / 47 \mathrm{mV}$ (Fig. 5c(i)) in the two channels, while the third probe 232 simultaneously recorded from another cell with amplitude of $62 \mathrm{mV}$ (Fig. 5c(ii)). Comparison of 233 the time derivatives (Fig. 5c(iii)) showed no discernible delay in the paired channels, while there 234 was a ca. $6 \mathrm{~ms}$ delay between paired and single probes. This delay time and probe separation 235 yield a signal propagation speed of $\sim 5.8 \mathrm{~cm} / \mathrm{s}$, which agrees with that reported in the literature ${ }^{31}$. 236 Overlay of the action potentials (Supplementary Fig. 14c,d) shows good agreement in the rising 
237 phase, and small deviations in the repolarization phase which can be attributed to different 238 changes in the two U-NWFET/cell junctions as a result of mechanical contraction ${ }^{32}$.

239 Third, paired U-NWFETs containing one ca. $50 \mathrm{~nm}$ FET with $0.75 \mu \mathrm{m}$ ROC and one ca. $2402000 \mathrm{~nm}$ FET with $1.5 \mu \mathrm{m}$ ROC on a single probe arm with $2 \mu \mathrm{m}$ separation were fabricated and 241 brought into contact with a HiPSC-CM (Fig. 5d). The channel with the ca. $0.75 \mu \mathrm{m}$ ROC, $50 \mathrm{~nm}$ 242 FET measured waveforms of ca. $50 \mathrm{mV}$ characteristic of intracellular cardiac action potentials 243 (Fig. 5d(i)), while the channel with the $2.0 \mu \mathrm{m}$ ROC, ca. $2000 \mathrm{~nm}$ FET measured sharp 244 downward spikes of ca. $2 \mathrm{mV}$ and $<5 \mathrm{~ms}$ duration characteristic of extracellular cardiac action 245 potentials (Fig. 5d(ii)). Close examination of the rising phase and derivative of the intracellular 246 trace in comparison to the extracellular trace (Fig 5d(iii)), shows that the measured extracellular 247 signal is dominated by a downward peak at the same time as the upwards phase of the action 248 potential. Extracellular waveforms measured using metal electrodes show increasing potential 249 from capacitive coupling with the intracellular space, which should be aligned with the time 250 derivative of the intracellular signal ${ }^{28,33}$, followed by a decrease from inward $\mathrm{Na}^{+}$current during 251 action potential firing ${ }^{33}$. Our observed monophasic negative waveform suggests the small size of 252 our recording element decreases capacitive coupling with the interior of the cell so that only 253 local potential changes derived from inward $\mathrm{Na}^{+}$currents are observed. Furthermore, previous 254 reports of simultaneous extracellular and intracellular measurement required separate patch 255 clamp and metal electrode array recording systems which can introduce complications such as 256 spatial mismatch of recording sites and difficulty in temporal synchronization ${ }^{28,33}$. In comparison, 257 our multiplexed measurement strategy provides more localized information to correlate 258 extracellular and intracellular action potentials. 
Last, we demonstrate the scalability of our U-NWFET devices by fabricating six device

260

261

262

263

264

265

266

267

268

269

270

271

272

273

274

275

276

277

278

279

280

281

regions containing 135 working single or multi U-NWFET devices (out of 168 addressable device sites) on a $76 \mathrm{~mm}$ wafer (Supplementary Fig. 15a,b). For typical cell experiments, the wafer is subsequently 'diced' to yield six chips that are individually mounted on printed circuit boards (Supplementary Fig. 5a). Separate measurements from HiPSC-CMs for each of the 6 chip device arrays yielded intracellular signal from at least 8 devices for each chip (Supplementary Fig. 15c). In one device array, we designed a series of probes containing 1-4 nanowires per probe arm (Supplementary Fig. 16a,b), and show simultaneous recording of action potentials in 10 channels from 4 separate cells (Supplementary Fig. 16c). These experiments validate the potential for using our U-NWFET probes for multiplexed recording to study the electrophysiology of cell networks.

\section{Conclusions}

In summary, we have demonstrated that the scalable ultrasmall U-NWFET probe arrays fabricated using deterministic shape-controlled nanowire assembly and selective spatiallydefined solid-state semiconductor-to-metal transformation have the capability to record full amplitude intracellular action potentials from primary neurons and other electrogenic cells, and the capacity for multiplexed recordings. These new studies complement other efforts from several groups focused on developing solid-state nanodevices for cell electrophysiology. While our number of recording channels is limited compared to hundreds of channels demonstrated in electrode array-based strategies ${ }^{12,14}$, it is notable that our U-NWFETs show the capability to record full-amplitude intracellular actions potentials that are similar to patch-clamp recordings, but now in a scalable format. This flexible device structure/fabrication approach has further provided direct information about the relationship between recording amplitude as a function of 
282 device curvature and size, and thus supports the developing "curvature hypothesis" relating 283 nanotopography to endocytosis and cytoskeleton dynamics ${ }^{17}$. One key challenge, encountered 284 both by U-NWFETs and other nanodevices, is the long-term stability of the intracellular 285 recording. We hypothesize that future studies exploring either (1) chemical anchoring via surface 286 functionalization with groups that can bind to the actin cytoskeletal localized near highly curved 287 membranes ${ }^{17,18,25}$ or (2) physical anchoring using spicule mesostructures ${ }^{34}$ and/or modulation of 288 nanowire morphology ${ }^{29}$ to increase the physical detachment force could improve the stability of 289 the intracellular device configuration. Supporting the potential of these proposed directions, 290 previous experiments indicate that functionalized free-standing nanowires can remain in the 291 interior of neurons for at least several hours ${ }^{35}$. More generally, this deterministic nanowire-based 292 fabrication strategy can be incorporated into other platforms, such as free-standing probes ${ }^{16}$, 293 which allow precise targeting of individual cells or subcellular structures, and mesh 294 electronics ${ }^{36,37}$ for in-vivo measurements. 


\section{References}

296 1. Chen, R., Canales, A. \& Anikeeva, P. Neural recording and modulation technologies. Nature Rev. 297 Mater. 2, 16093 (2017).

298 2. Spira, M.E. \& Hai, A. Multi-electrode array technologies for neuroscience and cardiology. Nature Nanotech. 8, 83-94 (2013).

$300 \quad 3 . \quad$ Kruskal, P.B., Jiang, Z., Gao, T. \& Lieber, C.M. Beyond the patch clamp: nanotechnologies for 301 intracellular recording. Neuron 86, 21-24 (2015).

302

4. Savtchenko, L.P., Poo, M.M. \& Rusakov, D.A. Electrodiffusion phenomena in neuroscience: a

303

304 neglected companion. Nature Rev. Neurosci. 18, 598 (2017).

305

5. Hamill, O.P., Marty, A., Neher, E., Sakmann, B. \& Sigworth, F.J. Improved patch-clamp techniques for high-resolution current recording from cells and cell-free membrane patches. Pflug. Arch. Eur. J. Phy. 391, 85-100 (1981).

6. Martina, M. \& Taverna, S. Patch-clamp methods and protocols, Edn. 2nd. (Humana Press, New York; 2014).

7. Jiang, Y.W. et al. Rational design of silicon structures for optically controlled multiscale biointerfaces. Nature Biomed. Eng. 2, 508-521 (2018).

8. Parameswaran, R. et al. Photoelectrochemical modulation of neuronal activity with free-standing coaxial silicon nanowires. Nature Nanotech. 13, 260-266 (2018).

9. Abbott, J., Ye, T.Y., Ham, D. \& Park, H. Optimizing nanoelectrode arrays for scalable intracellular electrophysiology. Accounts Chem. Res. 51, 600-608 (2018).

10. McGuire, A.F., Santoro, F. \& Cui, B.X. Interfacing cells with vertical nanoscale devices: applications and characterization. Annu. Rev. Anal. Chem. 11, 101-126 (2018).

11. Spira, M.E., Shmoel, N., Huang, S.H.M. \& Erez, H. Multisite attenuated intracellular recordings by extracellular multielectrode arrays, a perspective. Front. Neurosci. 12, 212, (2018).

12. Abbott, J. et al. CMOS nanoelectrode array for all-electrical intracellular electrophysiological imaging. Nature Nanotech. 12, 460-466 (2017).

13. Dipalo, M. et al. Intracellular and extracellular recording of spontaneous action potentials in mammalian neurons and cardiac cells with 3D plasmonic nanoelectrodes. Nano Lett. 17, 39323939 (2017).

14. Dipalo, M. et al. Plasmonic meta-electrodes allow intracellular recordings at network level on high-density CMOS-multi-electrode arrays. Nature Nanotech. 13, 965-972 (2018).

15. Tian, B.Z. et al. Three-dimensional, flexible nanoscale field-effect transistors as localized bioprobes. Science 329, 830-834 (2010).

16. Qing, Q. et al. Free-standing kinked nanowire transistor probes for targeted intracellular recording in three dimensions. Nature Nanotech. 9, 142-147 (2014).

330 17. Lou, H.Y., Zhao, W.T., Zeng, Y.P. \& Cui, B.X. The role of membrane curvature in nanoscale topography-induced intracellular signaling. Accounts of Chem. Res. 51, 1046-1053 (2018).

332 18. Zhao, W.T. et al. Nanoscale manipulation of membrane curvature for probing endocytosis in live cells. Nature Nanotech. 12, 750-756 (2017). 
19. Iversen, L., Mathiasen, S., Larsen, J.B. \& Stamou, D. Membrane curvature bends the laws of physics and chemistry. Nature Chem. Biol. 11, 822-825 (2015).

20. Kaksonen, M. \& Roux, A. Mechanisms of clathrin-mediated endocytosis. Nature Rev. Mol. Cell Bio. 19, 313-326 (2018).

21. Zhao, Y. et al. Shape-controlled deterministic assembly of nanowires. Nano Lett. 16, 2644-2650 (2016).

22. Wu, Y., Xiang, J., Yang, C., Lu, W. \& Lieber, C.M. Single-crystal metallic nanowires and metal/semiconducotor nanowire heterostructures. Nature 430, 61-65 (2004).

23. Study, R. E., \& Kral, M. G. Spontaneous action potential activity in isolated dorsal root ganglion neurons from rats with a painful neuropathy. Pain 65, 235-242 (1996).

24. Delmas, P., Hao, J., \& Rodat-Despoix, L. Molecular mechanisms of mechanotransduction in mammalian sensory neurons. Nature Rev. Neurosci. 12, 139-153 (2011).

25. Aalipour, A., Xu, A.M., Leal-Ortiz, S., Garner, C.C. \& Melosh, N.A. Plasma membrane and actin cytoskeleton as synergistic barriers to nanowire cell penetration. Langmuir 30, 12362-12367 (2014).

26. Cossell, L. et al. Functional organization of excitatory synaptic strength in primary visual cortex. Nature 518, 399-403 (2015).

27. Burridge, P.W. et al. Chemically defined generation of human cardiomyocytes. Nature Methods 11, 855-860 (2014).

28. Sanders, K.M., Ward, S.M. \& Hennig, G.W. Problems with extracellular recording of electrical activity in gastrointestinal muscle. Nature Rev. Gastro. Hepat. 13, 731-741 (2016).

29. Dipalo, M. et al. Cells adhering to 3D vertical nanostructures: cell membrane reshaping without stable internalization. Nano Lett. 18, 6100-6105 (2018).

30. Dietschy, J. M., \& Turley, S. D. Thematic review series: brain Lipids. Cholesterol metabolism in the central nervous system during early development and in the mature animal. J. Lipid Res 45, 1375-1397 (2004).

31. Zhu, H.Q. et al. Two dimensional electrophysiological characterization of human pluripotent stem cell-derived cardiomyocyte system. Sci. Rep. 7, 43210 (2017).

32. Woodcock, E. A., \& Matkovich, S. J. Cardiomyocytes structure, function and associated pathologies. Int. J. Biochem. Cell Biol, 37, 1746-1751 (2005).

33. Gold, C., Henze, D.A., Koch, C. \& Buzsáki, G. On the origin of the extracellular action potential waveform : a modeling study. J. Neurophysiol. 95, 3113-3128 (2006).

34. Luo, Z. et al. Atomic gold - enabled three-dimensional lithography for silicon mesostructures. Science, 348, 1451-1455 (2015).

35. Lee, J.H., Zhang, A.Q., You, S.S. \& Lieber, C.M. Spontaneous internalization of cell penetrating peptide-modified nanowires into primary neurons. Nano Lett. 16, 1509-1513 (2016).

36. Fu, T.M. et al. Stable long-term chronic brain mapping at the single-neuron level. Nature Methods 13, 875-882 (2016).

37. Hong, G.S. et al. A method for single-neuron chronic recording from the retina in awake mice. Science 360, 1447-1451 (2018). 


\section{Acknowledgements}

376 C.M.L. acknowledges support from Air Force Office of Scientific Research (FA9550-14-1377 0136). S.S.Y acknowledges an NSF Graduate Research Fellowship. This work is performed in 378 part at the Center for Nanoscale Systems (CNS) of Harvard University.

379

380 Author contributions

381 Y.Z., S.S.Y. and C.M.L. conceived and designed the experiments, Y.Z., S.S.Y., and A.Z. 382 performed the experiments and analyzed the data, Y.Z., S.S.Y., A.Z and C.M.L. co-wrote the 383 paper. All authors discussed the results and commented on the manuscript.

385 Additional information

386 Supplementary information is available in the online version of the paper. Reprints and 387 permissions information is available online at www.nature.com/reprints. Correspondence and 388 requests for materials should be addressed to C.M.L (cml@cmliris.harvard.edu $)$.

389

390 Competing financial interests

391 The authors declare no competing financial interests. 


\section{Figure captions:}

393

394

395

396

397

398

399

400

401

402

403

404

405

406

407

408

409

410

411

412

413

414

415

416

417

418

419

420

421

Figure 1 | Ultrasmall U-shaped nanowire field effect transistor probe as a new approach for electrophysiology. a, Schematics of intracellular recording by a U-NWFET probe. The location, size, geometry of each probe and the sensor size can be well modulated by deterministic shapecontrolled nanowire transfer technique and spatially-defined transformation of $\mathrm{Si}$ nanowire segments to $\mathrm{NiSi}$, respectively. b, Schematics of two possible probe-cell interfaces: (i) Internalization and high resistance seal of short-channel U-NWFET to the cell membrane enables high amplitude recording. The sensitive p-type Si NWFET region and the metallic NiSi region on U-shaped nanowire are marked with red and silver respectively. Nanowire is shown modified with phospholipid. (ii) Partial sealing/internalization of U-NWFET with longer channel length/ROC results in attenuated intracellular-like action potential recording.

\section{Figure 2 | Fabrication and characterization of U-shaped nanowire field effect transistor} probes. a, Schematics of device fabrication. (i) Assembly of U-shaped nanowires devices on Ni sacrificial layer and bottom $\mathrm{Si}_{3} \mathrm{~N}_{4}$ passivation layer, and electrical contacts to the transferred Ushaped nanowires are made via deposition of $\mathrm{Cr} / \mathrm{Au} / \mathrm{Cr}(1.5 / 60 / 60 \mathrm{~nm})$ metal interconnects, where the relative $\mathrm{Cr} / \mathrm{Au} / \mathrm{Cr}$ thicknesses yield a built-in strain that bends up the probe upon release;(ii) Deposition of top $\mathrm{Si}_{3} \mathrm{~N}_{4}$ passivation layer and $\mathrm{Ni}$ diffusion layer followed by rapid thermal annealing to transform the Si nanowire segments underneath and adjacent to the $\mathrm{Ni}$ diffusion layer to NiSi, and thus generate a local FET at the tip of the U-shaped nanowire; (iii) Probes bending upward after etching the Ni diffusion and sacrificial layers. b, Optical image of three devices following deposition of metal interconnects and before deposition of nickel diffusion layer. Inset is the zoom-in view showing that a U-shaped nanowire is deterministically transferred to the device tip. Scale bar $20 \mu \mathrm{m}$. c, SEM image of device after Ni diffusion. Scale bar $500 \mathrm{~nm}$. Inset is a zoom-in SEM image of the dashed region showing the resulting local FET at the U-shaped nanowire tip. Scale bar $50 \mathrm{~nm}$. Imaging with backscattered electrons (BSE) shows the Si (dark region) and NiSi (bright region) distribution on the U-shaped nanowire. Scale bar $50 \mathrm{~nm}$. d, Optical image of bend-up device array in water. Scale bar $20 \mu \mathrm{m}$. e-g, Current versus drain-source voltage $\left(\mathrm{V}_{\mathrm{ds}}\right)$ traces for 10 devices in the dry state for (e) ca. $50 \mathrm{~nm}$, (f) ca. $500 \mathrm{~nm}$, and (g) ca. $2000 \mathrm{~nm}$ channel lengths. Insets show SEM images taken using BSE mode of the local FET following removal of Ni layer. Scale bars: (e) $50 \mathrm{~nm}$, (f) $0.5 \mu \mathrm{m},(\mathbf{g}) 0.5 \mu \mathrm{m}$. h- 
422 j, (Left) Conductance versus reference water-gate potential $\left(\mathrm{V}_{\mathrm{g}}\right)$ recorded from one 423 representative device for each channel length ((h) ca. $50 \mathrm{~nm}$, (i) ca. $500 \mathrm{~nm}$, and (j) ca. $2000 \mathrm{~nm})$ 424 in Tyrode's solution. (Right) Box and whisker plot showing distribution (N=10) of 425 transconductances for devices of each channel length gated by a reference electrode in Tyrode's 426 solution. The blue triangle indicates the mean of the transconductances and the top and bottom 427 edges of the box indicate the upper/lower quartiles respectively. The error bars reflect \pm 1 s.e.m.

428

429

430

431

432

433

434

435

436

437

438

439

440

441

442

443

444

445

446

447

448

449

450

451

Figure 3 | Intracellular recording of dorsal root ganglion neurons neuron by ultrasmall Ushaped nanowire field effect transistor probe. a, Intracellular action potentials recorded sequentially from 6 independent neurons using the same U-NWFET probe without remodification. The numbering of the cells indicates the order in which the measurements occurred. The probe has ca. $50 \mathrm{~nm}$ FET channel length and $0.75 \mu \mathrm{m}$ ROC. b, The summary of recorded action potentials from the 6 different cells in panel a in chronological order. Maximum signal amplitude remains similar between these measurements, showing that the probe is highly reusable. The signal-to-noise of these recordings is $115 \pm 29(\mathrm{~N}=6)$. c, Intracellular recording trace from a cell with regular firing pattern showing a gradual increase in baseline potential and decrease in action potential amplitude. d, Representative trace from a cell with irregular firing pattern where subthreshold activities appear in regions outlined by blue dashed lines. e, Zoom in regions of three different areas from panel $\mathbf{d}$ showing different types of subthreshold activities, highlighted by the red arrows and blue dashed regions.

\section{Figure 4 | Effect of size and geometry of U-shaped nanowire field effect transistor probes} on electrophysiological recordings. a, Optical image of U-NWFET probes with different ROC before deposition of Ni diffusion layer (i) $0.75 \mu \mathrm{m}$, (ii) $1 \mu \mathrm{m}$, (iii) $1.5 \mu \mathrm{m}$ and (iv) $2 \mu \mathrm{m}$. b,c, Intracellular/intracellular-like recording from DRG neuron by ca. $50 \mathrm{~nm}$ FET channel length probe with (b) $1 \mu \mathrm{m}$ and (c) $1.5 \mu \mathrm{m}$ ROC. Insets are the zoom-in view of selected action potentials. d-e, Plot of maximum recorded spike amplitude of recorded action potentials from DRG neurons (d) and HiPSC-CMs (e) versus ROC with fixed ca. 50 nm FET length. Coloured bars indicate the maximum spike amplitudes measured in the given data set. The statistical significance shown above obtained by comparing the datasets below the ends of the black line using the student's t-test. ****: p-value $<0.0001$. The blue letters 'b', 'c' and 'i' highlight the data points from panels b,c,i. f, Scatter plot of maximum recorded spike amplitude of DRG 
neurons (i) and HiPSC-CMs (ii) for ca. $500 \mathrm{~nm}$ FET channel lengths with $0.75 \mu \mathrm{m}$ ROC. The statistical significance $* * * *$ shown in (i) and (ii) obtained by comparing the datasets in (i) and (ii) with datasets of ROC $0.75 \mu \mathrm{m}$ in (d) and (e) respectively. g, Plot of maximum recorded spike amplitude of HiPSC-CMs with ca. $2000 \mathrm{~nm}$ FET channel. Blue circles represent intracellular data recorded from U-NWFETs with $1.0 \mu \mathrm{m}$ ROC and yellow circles represent intracellular data recorded from U-NWFET with $1.5 \mu \mathrm{m}$ ROC. Red triangles represent extracellular data recorded from U-NWFETs with $1.5 \mu \mathrm{m}$ ROC. The letter $\mathrm{h}$ represents the data point for the trace shown in panel h. Note that for all scatter plots, ca. 30 measurements were attempted for each U-NWFET channel length and ROC, and recordings which did not result in measurement of intracellular/intracellular-like or extracellular action potentials were considered as $0 \mathrm{mV} \max$ spike amplitude and not shown for clarity but are summarized in Supplementary Table 3. h, Extracellular recording from HiPSC-CMs by ca. $2000 \mathrm{~nm}$ FET channel length probe with $1.5 \mu \mathrm{m}$ ROC. Inset is the zoom-in of the highlighted spike. i, Intracellular recording from HiPSC-CMs by ca. $50 \mathrm{~nm}$ FET channel length probe with $0.75 \mu \mathrm{m}$ ROC.

\section{Figure 5 | Multiplexed electrophysiological recording by U-shaped nanowire field effect} transistor probes. a, Schematics of (i) simultaneous multisite intracellular recording from single neuron by paired U-NWFETs on one probe arm, (ii) multiplexed intracellular recording from different cells by U-NWFETs on different probe arms, and (iii) simultaneous intracellular/extracellular recording from one cell by paired U-NWFETs on one probe arm. b, (i) simultaneous intracellular recording from one DRG neuron by two ca. $50 \mathrm{~nm}$ FETs with $0.75 \mu \mathrm{m}$ ROC on one probe arm with a $2 \mu \mathrm{m}$ separation. (ii) the derivative of traces in dashed block marked region. The vertical dashed guiding line indicates the time point of the first action potential. No time delay is observed. c, multiplexed intracellular recording from two HiPSCCMs by (i) one paired U-NWFET probe and (ii) one single U-NWFET probe. (iii) the derivative of marked region. Two probes arms are fabricated with a distance of $350 \mu \mathrm{m}$. d, simultaneous intracellular/extracellular recording from one HiPSC-CM by (i) one ca. $50 \mathrm{~nm}$ FET with $0.75 \mu \mathrm{m}$ ROC (red top, original intracellular signal; red bottom, derivative of intracellular signal) and (ii) one ca. $2000 \mathrm{~nm}$ FET with $1.5 \mu \mathrm{m}$ ROC on one probe arm with $2 \mu \mathrm{m}$ separation. (iii) Closer examination of the marked region. 
483 Nanowire synthesis

484 Si nanowires (p-type) with $15 \mathrm{~nm}$ diameters were synthesized by a gold nanocluster-catalyzed vapourliquid-solid growth method ${ }^{21}$. Growth substrates $\left(15 \times 60 \mathrm{~mm}^{2}\right.$ pieces of Si wafer with $600 \mathrm{~nm}$ thermal oxide, Nova Electronic Materials, Flower Mound, TX) were oxygen plasma cleaned (100 W, 2 min, 50 cubic centimetres per minute $(\mathrm{sccm}) \mathrm{O}_{2}$; PJ Plasma Surface Treatment System, Billerica, MA), treated with poly-L-lysine solution $(0.1 \%, 150,000-300,000 \mathrm{~g} / \mathrm{mol}$, Ted Pella, Inc., Redding, CA) for $5 \mathrm{~min}$, rinsed thoroughly with deionized (DI) water and dried with nitrogen. Then $1 \mathrm{~mL}$ of aqueous solution of $10 \mathrm{~nm}$ gold nanoparticles (Ted Pella, Inc., Redding, CA) with concentration of $1.9 \times 10^{12} /$ particles $/ \mathrm{mL}$ was dispersed ${ }^{38}$ on the substrate for 2 min followed by thorough rinsing with DI water and drying with nitrogen (gold nanoparticle surface concentration: $0.01-0.04$ particles $/ \mu \mathrm{m}^{2}$ ). The substrate was then placed into a home-built chemical vapour deposition reactor and the system was evacuated to a base pressure of $0.6 \mathrm{mTorr}$. Nanowires were synthesized at $430{ }^{\circ} \mathrm{C}$ at a total pressure of 40 Torr with gas flow rates of $2.5 \mathrm{sccm}$ silane $\left(\mathrm{SiH}_{4}, 99.9999 \%\right.$, Voltaix, Branchburg, $\left.\mathrm{NJ}\right)$ as the silicon reactant, $3.1 \mathrm{sccm}$ diborane $\left(\mathrm{B}_{2} \mathrm{H}_{6}, 100\right.$ p.p.m. in $\mathrm{H}_{2}$, Voltaix, Branchburg, $\left.\mathrm{NJ}\right)$ as the p-type dopant, and 60 sccm hydrogen $\left(\mathrm{H}_{2}, 99.999 \%\right.$; Matheson, Basking Ridge, $\left.\mathrm{NJ}\right)$ as the carrier gas. Typical growth times of $1 \mathrm{hr}$ yielded nanowires with average lengths of ca. $50 \mu \mathrm{m}$.

U-NWFET probe array fabrication

500 Key steps involved in the fabrication of U-NWFET probe arrays are shown in Fig. 2 and Supplementary 501 Fig. 1, with the key parameters as follows:

502 (1) LOR 3A (Microchem, Westborough, MA) and diluted S1805 (S1805: Thinner-P = 1:2 (vol : vol), 503 Microchem, Westborough, MA) were spin-coated on a $\mathrm{Si}_{3} \mathrm{~N}_{4} / \mathrm{SiO}_{2}$-coated $\mathrm{Si}$ wafer (200 $\mathrm{nm} \mathrm{Si}_{3} \mathrm{~N}_{4}, 100$ $504 \mathrm{~nm} \mathrm{SiO} 2$ on p-type $\mathrm{Si}, 0.005 \Omega \mathrm{cm}$, or $600 \mathrm{~nm}$ thermal $\mathrm{SiO}_{2}$ on n-type $\mathrm{Si}, 0.005 \Omega \mathrm{cm}$, Nova Electronic 505 Materials, Flower Mound, TX) and baked at $180{ }^{\circ} \mathrm{C}$ for $5 \mathrm{~min}$ and at $115^{\circ} \mathrm{C}$ for $1 \mathrm{~min}$ respectively. The 506 photoresist was patterned by photolithography (PL) with a Maskless aligner (MLA150, Heidelberg, Germany) and developed (MF-CD-26, MicroChem Corp., Newton, MA) for 30s. Following this PL process, a 60-nm thick Ni sacrificial layer was deposited by thermal evaporation (Sharon Vacuum Co. Inc., Brockton, MA), followed by a lift-off step (Remover PG, MicroChem Corp., Newton, MA). 511 NWFET probe: $30 \mu \mathrm{m}$ x $100 \mu \mathrm{m}$ for single U-NWFET probes (Supplementary Fig. 1h(i)) or $90 \mu \mathrm{m} \times 100$ $512 \mu \mathrm{m}$ for up to 4 U-NWFETs probes per bend-up probe arm (Supplementary Fig. 1h(ii)).

513 (2) The PL process in step 1 was repeated to define an $80 \mu \mathrm{m}$ long bottom region for sputter deposition of $51460 \mathrm{~nm}$ of $\mathrm{Si}_{3} \mathrm{~N}_{4}$ passivation layer (Orion 3 Sputtering Systems, AJA International Inc., Scituate, MA). The 
main body of $\mathrm{Si}_{3} \mathrm{~N}_{4}$ passivation layer ( $75 \mu \mathrm{m}$ long) was deposited on the Ni sacrificial layer with $5 \mu \mathrm{m}$ $\mathrm{Si}_{3} \mathrm{~N}_{4}$ extending outside of the sacrificial region. (Supplementary Fig. 1b)

(3) LOR 1A (Microchem, Westborough, MA) and diluted S1805 (S1805: Thinner-P = 1:2 (vol : vol)) was spin-coated and baked at $180{ }^{\circ} \mathrm{C}$ for $5 \mathrm{~min}$ and at $115^{\circ} \mathrm{C}$ for $1 \mathrm{~min}$, respectively. Then the PL process in step 1 was repeated for defining arrays of trenches with shapes and ROCs as described in the main text. (Supplementary Fig. 1c)

(4) Shape-controlled deterministic nanowire assembly was used to align disordered straight nanowires into U-shaped arrays as described previously ${ }^{21}$. (Supplementary Fig. 1d) Briefly, a wafer with an array of trenches was mounted onto a micromanipulator-controlled moveable stage, covered with mineral oil (viscosity $v \approx 70 \mathrm{mPa} \bullet \mathrm{s}, \# 330760$, Sigma-Aldrich, St. Louis, MO) as the lubricant, and then the nanowire growth substrate was brought into contact with the target substrate with controlled contact pressure. The target substrate was moved at a constant velocity of ca. $5 \mathrm{~mm} / \mathrm{min}$ with respect to the fixed nanowire growth substrate, and then the growth substrate was removed, and the target substrate rinsed with octane (98\%, Sigma-Aldrich, St. Louis, MO) to remove the lubricant. Estimations of the U-shaped nanowire strain are calculated and shown in Supplementary Table 2.

(5) Aluminium oxide $\left(\mathrm{Al}_{2} \mathrm{O}_{3}\right)$ was deposited directly after the nanowire assembly by atomic layer deposition (ALD, S200, Cambridge NanoTech, Waltham, MA) with 1 cycle $(1.4 \AA)$ at $80{ }^{\circ} \mathrm{C}$ to fix the Ushaped nanowires on the bottom passivation layer and then all photoresist was removed in Remover PG. (Supplementary Fig. 1e)

(6) Step 1 was repeated for simultaneously patterning electrical interconnects to the U-NWFET as well as connects to large pads used as the input/output (I/O) region. Native oxide on the nanowire was etched by buffered oxide etch (BOE, 7:1, Microchem, Westborough, MA) for 10s before thermal deposition of asymmetrically strained metal $\mathrm{Cr} / \mathrm{Au} / \mathrm{Cr}(1.5 / 60 / 60 \mathrm{~nm})$. The strained metal leads the U-NWFET probe to bend off the wafer surface following etching of the sacrificial layer, like that described in previous work $^{21}$.

(7) Step 2 was repeated to deposit $60 \mathrm{~nm}$ of $\mathrm{Si}_{3} \mathrm{~N}_{4}$ as electrical passivation over exposed metal features except for the I/O pad region. (Fig. 2b and Supplementary Fig. 1f).

(8) Electron-beam lithography (EBL) or PL was used to define the Ni diffusion region with shape and position as described in the main text. Specifically, EBL was used for U-NWFET probe with ca. $50 \mathrm{~nm}$ (Fig. 2c) and ca. $500 \mathrm{~nm}$ channel length (Supplementary Fig. 2c). For the EBL process, copolymer MMA (EL6, Microchem, Westborough, MA) and polymethyl methacrylate (PMMA, 950-C2, Microchem, Westborough, MA) were spin-coated and baked at $180^{\circ} \mathrm{C}$ for 5 min sequentially. The resists were then patterned with an EBL system (ELS-F125, Elionix, Japan) and developed (MIBK/IPA 1:1, MicroChem Corp., Newton, MA) for 60 s. For U-NWFET probe with ca. $2000 \mathrm{~nm}$ channel length (Supplementary 
Fig. 2d), the same PL process in step 1 can be used to define regions for Ni deposition. Native oxide on the nanowire was removed by BOE for 10 seconds before deposition of $20 \mathrm{~nm} \mathrm{Ni}$ via thermal evaporation. After lift-off, the chip was annealed by Rapid Thermal Processor (RTP, 600xp, Modular Process Technology, San Jose, CA) in forming gas $\left(\mathrm{H}_{2}: \mathrm{N}_{2} 10: 90\right)$ at $350{ }^{\circ} \mathrm{C}$ for $5 \mathrm{~min}$ to transform $\mathrm{Si}$ nanowire segments underneath and adjacent to $\mathrm{Ni}$ diffusion layer to nickel silicide ${ }^{22}$, thereby generating a localized sensing element. (Supplementary Fig. 1g)

(9) Polydimethylsiloxane (PDMS) was prepared by first pouring Sylgard 184 (Dow Corning, Inc, Midland, Michigan) elastomer mixed in a 10:1 ratio of base to curing agent into a petri dish, and then curing overnight at $55{ }^{\circ} \mathrm{C}$ in a convection oven. A PDMS chamber with ca. $20 \times 30 \mathrm{~mm}^{2}$ opening and ca. $0.5 \mathrm{~cm}$ sidewalls were cut from the cured PDMS and mounted around the device region using Kwik-Sil silicone adhesive (World Precision Instruments, Sarasota, FL). A printed circuit board (PCB, UXCell, Hong Kong, China) connector was then mounted adjacent to the $\mathrm{I} / \mathrm{O}$ region of devices and then wirebonded to the U-NWFET probe I/O pads (Supplementary Fig. 5a). Probes were kept in dry-keeper desiccator cabinet (H-B Instrument-Bel-Art, Wayne, NJ). Before electrical characterizations and/or electrophysiological measurements, the Ni sacrificial layers and remaining $\mathrm{Ni}$ from the diffusion layer were removed in nickel etchant (Nickel Etchant TFB, Transense, Danvers, MA) for 3 - 5mins, which allowed release of these devices into three-dimensional bend-up structure (Fig. $2 \mathrm{~d}$ and Supplementary Fig. 1h). Following release, the devices were rinsed in DI water 5-10 times for $20 \mathrm{~s}$ each.

\section{Device Characterization}

Overview optical images of the measurement set-up and U-NWFET probe chip to instrument I/O were acquired with an SLR digital camera (Canon U.S.A., Inc., Melville, NY) and higher resolution brightfield optical images of individual U-NWFET probes and probe arrays were acquired by Olympus BX50WI with Andor Luca EMCCD camera. High-resolution SEM images of nanowires, including intermediate fabrication steps, were acquired by Zeiss Ultra Plus field emission SEM (Carl Zeiss, Germany). A backscattered electron (BSE) detector was used to obtain high-resolution compositionsensitive maps based on the electron elastic scattering difference of atomic number on the sample. UNWFET fabricated for characterization did not have Ni sacrificial layer to improve contrast during SEM imaging. The BSE images show the silicon and nickel silicide segments on U-shaped nanowires: the bright region indicates nickel/nickel silicide, and dark region indicates p-type Si.

For electrical characterization, one arm of the U-NWFET is considered as the source, and the other arm is considered as the drain. A voltage, $\mathrm{V}_{\mathrm{ds}}$ was applied between the source and drain of the U-NWFET, and the resulting current, $I_{d s}$ was measured. The electrical conductance $\left(I_{d s} / V_{d s}\right)$ of the U-NWFET devices were measured in the dry state by sweeping $\mathrm{V}_{\mathrm{ds}}$ between -1 and $1 \mathrm{~V}$ and measuring $\mathrm{I}_{\mathrm{ds}}$ using a homemade battery powered 16-channel current preamplifier with bandwidth of $6 \mathrm{kHz}$ which amplified the current 
signal for recording using a 16-channel A/D converter (Axon Digidata 1440A; Molecular Devices, San Jose, CA) controlled by pCLAMP 10.7 software (Molecular Devices). The $\mathrm{I}_{\mathrm{ds}}-\mathrm{V}_{\mathrm{ds}}$ date were recorded in 585 air/dry state.

\section{Surface Functionalization of U-NWFET probes}

587 Phospholipid vesicles were prepared for use to functionalized U-NWFET probes in the following manner 588 similar to previous papers ${ }^{15,16}$ : (1) 1,2-dimyristoyl-sn-glycero-3-phosphocholine (DMPC, Avanti Polar 589 Lipids Inc., Alabaster, Alabama) was suspended in chloroform (anhydrous, > 99\%, Sigma-Aldrich, St. 590 Louis, MO) to a concentration of $20 \mathrm{mg} / \mathrm{mL}$ and mixed with 1\% mass of 1-myristoyl-2-[12-[(7-nitro-2591 1,3-benzoxadiazol-4-yl)amino]dodecanoyl]-sn-glycero-3-phosphocholine (NBD-lipid, Avanti Polar 592 Lipids Inc. Alabaster, Alabama). (2) The solution of DMPC/NBD-lipid was then placed into a vacuum 593 desiccator for at least $6 \mathrm{hrs}$ to evaporate off the chloroform. (3) The resulting powder was resuspended in 594 phosphate buffered saline (1x PBS, HyClone, South Logan, Utah) to concentration of $1 \mathrm{mg} / \mathrm{mL}$ and the 595 lipid solution was placed in a water bath at $37{ }^{\circ} \mathrm{C}$ for at least $2 \mathrm{hrs}$ with periodic agitation using a vortex 596 mixer (30s every 20 minutes; Maxi Mix II, Barnstead/Thermolyne Corp, Dubuque TX) to ensure full 597 rehydration. (4) The resulting lipid solution was sonicated using a tip sonicator (25\% amplitude, $10 \mathrm{~s} / 15 \mathrm{~s}$ pulse on/off, Branson Ultrasonics Sonifier S-4501, Branson Ultrasonics, Milford, CT) at ca. $37{ }^{\circ} \mathrm{C}$ for 2 hrs. (5) Following sonication, the lipid solution was sterile filtered (0.2 $\mu \mathrm{m}$ Acrodisc syringe filter, PN 4192, Pall Corporation, Port Washington, New York), and used within 1 hr of preparation.

Immediately prior to measurements, U-NWFET probe arrays with a mounted PDMS chamber (Supplementary Fig. 5b) were incubated for $2 \mathrm{hr}$ in $1.5 \mathrm{~mL}$ of the prepared lipid vesicle solution to allow functionalization of U-NWFET as reported previously for other nanowire devices ${ }^{15,16}$. Following incubation, the U-NWFET probe arrays were rinsed in Tyrode's solution (in $\mathrm{mM}, \mathrm{NaCl} 155, \mathrm{KCl} 3.5$, $\mathrm{MgCl}_{2}$ 1, $\mathrm{CaCl}_{2}$ 1.5, HEPES 10, D-glucose 10, pH 7.4 for DRG neurons, or $\mathrm{NaCl} 138, \mathrm{KCl} 4, \mathrm{CaCl}_{2} 2$, $\mathrm{MgCl}_{2}$ 1, $\mathrm{Na}_{2} \mathrm{HPO}_{4}$ 0.33, HEPES 10, glucose 10, pH 7.4 for HiPSC-CMs, all chemicals in Tyrode's solution were purchased from Sigma-Aldrich, St. Louis, MO) 5-10 times for $20 \mathrm{~s}$, ca. $3 \mathrm{~mL}$ each.

\section{Device Characterization in Tyrode's Solution}

610 Following phospholipid modification, electrical measurements were carried out in Tyrode's solution. The electrical conductance of the U-NWFETs was continuously measured by recording the drain-source current $\left(\mathrm{I}_{\mathrm{ds}}\right)$ at a fixed source-drain DC bias between $0.1-0.2 \mathrm{~V}$ by the electronic measurement setup mentioned above. The sensitivities (transconductance) were then obtained by sweeping an $\mathrm{Ag} / \mathrm{AgCl}$ reference electrode (2.0 x 4.0 mm, E-201, Warner Instruments, Hamden, CT) between $-100 \mathrm{mV}$ and 100 $\mathrm{mV}$ and measuring the corresponding linear change in U-NWFET conductance. The measured average (in 10 samples) conductance of U-NWFET for channel length ca. $50 \mathrm{~nm}$, ca. $500 \mathrm{~nm}$, ca. $2000 \mathrm{~nm}$ are $3.3 \pm$ $0.6,0.7 \pm 0.2$ and $0.3 \pm 0.1 \mu \mathrm{S}$, with average sensitivity of $5.4 \pm 1.3,2.3 \pm 0.7$ and $0.9 \pm 0.3 \mu \mathrm{S} / \mathrm{V}$ 
respectively. An inverse relationship exists between conductance, and consequently transconductance, and channel length, as expected from the relationship: $(\mathrm{G}=\sigma \mathrm{A} / \mathrm{L})$, where $\mathrm{G}$ is the channel conductance, $\sigma$ is the electrical conductivity and $\mathrm{A}$ is the cross-sectional area of the wire and $\mathrm{L}$ is the channel length ${ }^{39}$.

To estimate the noise level of the U-NWFETs devices, the conductance of the 10 devices for each channel length was measured at using the $\mathrm{Ag} / \mathrm{AgCl}$ reference electrode to fix the solution voltage at 0 for ca. $5 \mathrm{~s}$. The standard deviation of the measured conductance was used to obtain the noise for each device in uS. Then, the transconductance of each device was used to convert the measured noise in uS into a value in $\mathrm{mV}$, and the resulting number is multiplied 3 to estimate the limit of detection as per convention. Averaging the 10 values for limit of detection (in $\mathrm{mV}$ ) for each channel length resulted in noise levels of $0.90 \pm 0.60 \mathrm{mV}, 1.2 \pm 0.9 \mathrm{mV}, 1.9 \pm 0.9 \mathrm{mV}$ for the ca. $50 \mathrm{~nm}, 500 \mathrm{~nm}$, and $2000 \mathrm{~nm}$ devices respectively.

\section{Preparation of flexible cell culture substrates}

A master mould for the culture substrate was first prepared by spin coating SU-8 2000.5 (Microchem, Westborough, MA) onto a Si wafer and patterning repeating $3 \mu \mathrm{m}$ wide lines with $3 \mu \mathrm{m}$ spacing using PL. After patterning, the master mould was hard baked on a hot plate at $180{ }^{\circ} \mathrm{C}$ for $2 \mathrm{hrs}$, and then silanized with tridecafluoro-1,1,2,2- tetrahydrooctyl-1-trichlorosilane (Sigma-Aldrich, St. Louis, MO) for 2 hrs in a vacuum desiccator, to enhance release of the PDMS template from the master mould ${ }^{40}$.

Flexible PDMS cell culture substrates were prepared by spin coating Sylgard 184 elastomer mixed in a 10:1 ratio of base to curing agent onto the master mould at $250 \mathrm{rpm}$ for $1 \mathrm{~min}$. The PDMS on the master mould was then cured in a convection oven set to $180^{\circ} \mathrm{C}$ for $2 \mathrm{hrs}$, resulting in a thickness of ca. $220 \mu \mathrm{m}$ and cut into pieces with size of ca. $10 \times 10 \mathrm{~mm}^{2}$ for cell culture. Prior to cell culture, PDMS substrates were autoclaved at $125^{\circ} \mathrm{C}$ for $1 \mathrm{hr}$, treated by $\mathrm{O}_{2}$ plasma $(100 \mathrm{~W}, 2 \mathrm{~min}, 50 \mathrm{sccm} \mathrm{O})$ and then washed in $75 \%$ (v/v) solution of ethanol (200 proof, KOPTEC; King of Prussia, PA)/water for $1 \mathrm{hr}$.

For DRG neuron culture, the PDMS was first functionalized with $40 \mu \mathrm{g} / \mathrm{ml}$ poly-D-lysine (Molecular weight $>300,000 \mathrm{~g} / \mathrm{mol}$, Sigma-Aldrich, St. Louis, MO) in DI water for $1 \mathrm{hr}$ at room temperature. After poly-D-lysine functionalization, PDMS was washed twice in DI water for $30 \mathrm{~s}$ each and air dried, and then was functionalized with $20 \mu \mathrm{g} / \mathrm{ml}$ laminin (Thermo Fisher Scientific, Waltham, MA) in Leibovitz's L-15 (Thermo Fisher Scientific, Waltham, MA) for $1 \mathrm{hr}$ at room temperature. Laminin solution was removed immediately before cell suspension was plated on PDMS.

For HiPSC-CM culture, the PDMS was functionalized sequentially with (1) $1 \%$ (3Aminopropyl)triethoxysilane (Sigma-Aldrich, St. Louis, MO) in $95 \%$ (v/v) solution of ethanol/DI water for 20 min at room temperature, followed by washing 3 times in ethanol for $30 \mathrm{~s}$ each, and 3 times in DI water for $30 \mathrm{~s}$ each; (2) $2.5 \%$ (v/v) Glutaraldehyde (Grade I, 50\% in $\mathrm{H}_{2} \mathrm{O}$, Sigma-Aldrich, St. Louis, $\mathrm{MO}$ )/water for $1 \mathrm{hr}$ at room temperature, followed by washing 3 times in DI water for 30s each, and (3) 
652 removed immediately before cell suspension was plated onto the PDMS.

\section{Cell culture}

654 Dissociated DRG cells were prepared as described previously ${ }^{35}$ and cultured in the $\mathrm{CO}_{2}$ incubator 655 overnight before use. Cells that can spontaneously fire (e.g., Supplementary Fig. 8) were selected for 656 recording. HiPSC-CMs were cultured as described in the NCardia online protocol ${ }^{41}$. Cryogenically frozen 657 Cor.4U HiPSC-CM vials (Cor.4U $\geq 250 \mathrm{k}$ cells, Ncardia Group, Cologne, Germany) were thawed in $37^{\circ} \mathrm{C}$ 658 water bath, and $0.5 \mathrm{~mL}$ of proprietary Cor.4U cell media (Ncardia group; Cologne, Germany) preheated 659 to $37^{\circ} \mathrm{C}$ was added to the vial. The cell solution was then homogenized by gentle aspiration and seeded at $66075 \mathrm{k}$ cells $/ \mathrm{cm}^{2}$ to achieve confluency onto the prepared PDMS substrates. Immediately following cell 661 seeding, the cell culture was left at room temperature for $20 \mathrm{~min}$ to allow the solution to settle and ensure an even distribution of cells. The cells were then cultured in a $5 \% \mathrm{CO}_{2}, 37{ }^{\circ} \mathrm{C}$ incubator and the Cor.4U cell media was first changed 6 hrs following plating. Subsequently, the media was changed every day and the cells were used within 2 weeks following seeding, once a uniformly contracting layer was observed. Electrophysiological recording with U-NWFET

The $\mathrm{Ag} / \mathrm{AgCl}$ reference electrode is used to fix the extracellular Tyrode's solution voltage to $0 \mathrm{~V}$ for cell measurements. A PDMS sheet with cultured DRG neurons or HiPSC-CMs was fixed upside down onto a homebuilt vacuum wand mounted on a $40 \mathrm{~nm}$ step resolution $\mathrm{x}-\mathrm{y}-\mathrm{z}$ micromanipulator (MP-285, Shutter Instruments) connected to a micromanipulator controller (MPC-200/ROE-200, Sutter Instruments) to position the cells over and bring the cells into contact with the U-NWFETs (Supplementary Fig. 5c). The

671 Tyrode's solution was maintained at room temperature for the DRG neuron experiments and at ca. $37{ }^{\circ} \mathrm{C}$

672 for the HiPSC-CM experiments. For longer (>3 minutes) HiPSC-CM intracellular recording

673 (Supplementary Fig. 9b, d), high pass filters were set to $0.4 \mathrm{~Hz}$, similar to that used by other groups $\mathrm{s}^{12,42,43}$.

\section{Patch-clamp recording}

675 Patch-clamp recording was performed at room temperature using Multiclamp 700B amplifier (Molecular 676 Devices) and Digidata 1440A Digitizer Acquisition System, controlled by pCLAMP 10.7 software 677 (Molecular Devices). Micropipettes were prepared using a micropipette puller (P-97, Sutter Instruments), 678 the pipette tip resistance ranged between 5-10 M 2 . DRG neurons were cultured on a glass coverslip with 679 the same modification as PDMS. Recording from DRG neurons was carried out in Tyrode's solution. The 680 micropipettes were filled with an internal solution consisting of (in mM): potassium L-aspartate 140, $681 \mathrm{NaCl} 13.5, \mathrm{MgCl}_{2}$ 1.8, ethylene glycol-bis(2-aminoethylether)-N,N,N',N'-tetraacetic acid (EGTA) 0.09, 682 HEPES 9, phosphocreatine di(tris) salt 14, adenosine 5'-triphosphate (ATP) magnesium salt 4, guanosine 
684 were purchased from Sigma-Aldrich, except GTP tris buffer, which was purchased from Thermo Fisher.

685

\section{Data availability}

687 The data that support the findings of this study are available from the corresponding author upon 688 reasonable request.

689

\section{References}

691 38. Patolsky, F., Zheng, G., \& Lieber, C. M. Fabrication of silicon nanowire devices for ultrasensitive,

692

693 label-free, real-time detection of biological and chemical species. Nature Protocols 1, 1711-1724 (2006).

694 39. Kittel, C. Introduction to solid state physics. 8th edn, (Wiley, 2005).

695 40. Minteer, S. D. Microfluidic techniques: reviews and protocols. Totowa, N.J., Humana Press. (2006)

696 41. NCardia, Cardiomyocytes User Manual, https://ncardia.com/files/documents/manuals/PluricyteCardiomyocyte_Manual_v2.pdf

698 42. Shmoel, Nava, et al. Multisite electrophysiological recordings by self-assembled loose-patch-like 699 junctions between cultured hippocampal neurons and mushroom-shaped microelectrodes. Scientific reports $\mathbf{6}, 27110$ (2016).

43. Xie, Chong, et al. Intracellular recording of action potentials by nanopillar electroporation. Nature Nanotech. 7, 185-190 (2012). 


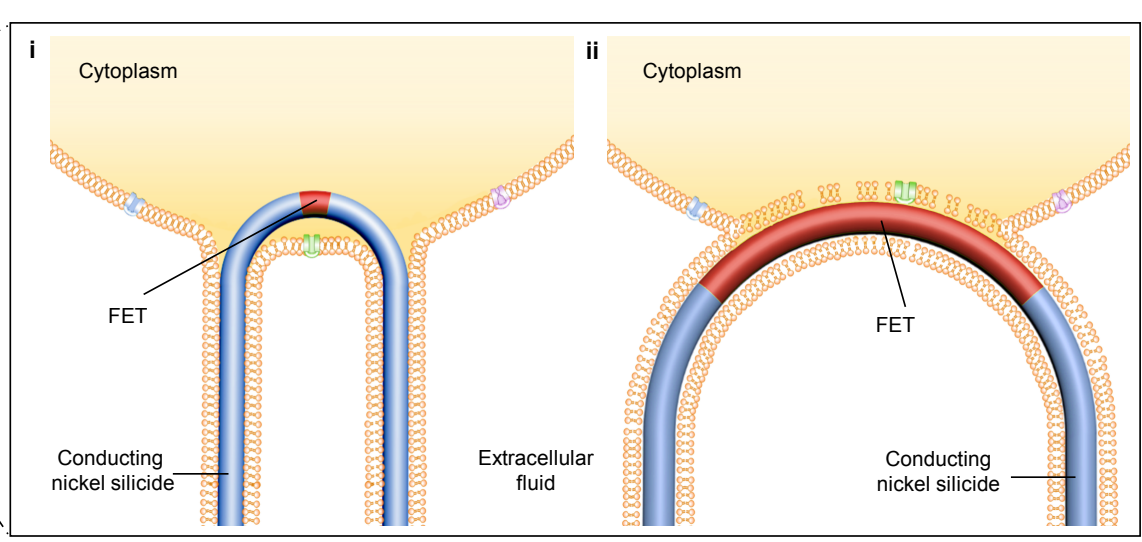


$\mathbf{a}$

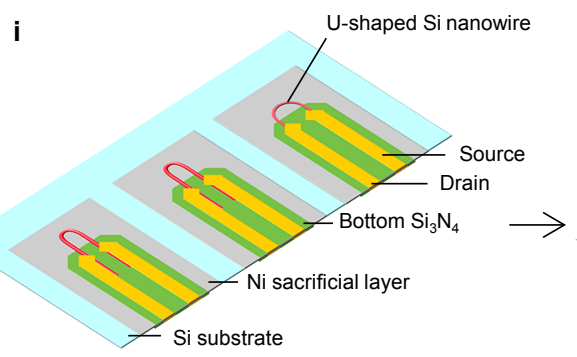

ii Ni diffusion layer

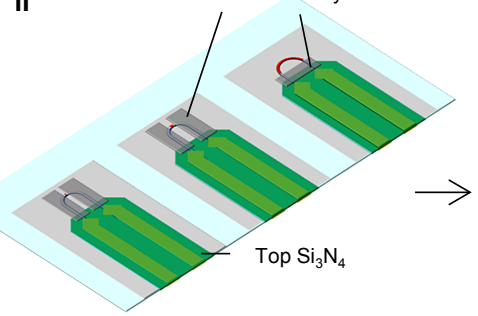

ii Bend-up nanoFET with modulated size

b

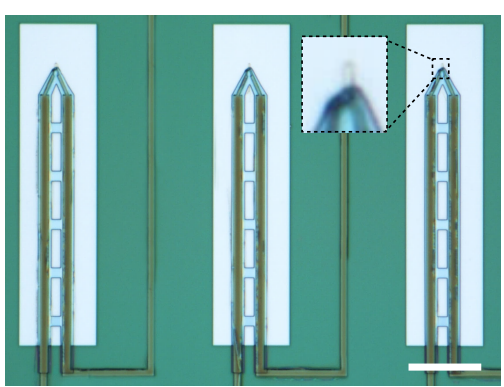

e 5000

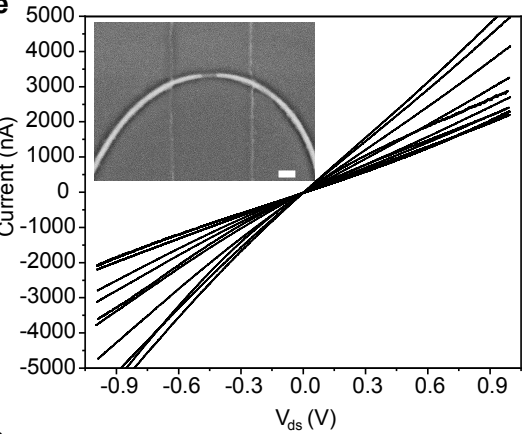

h

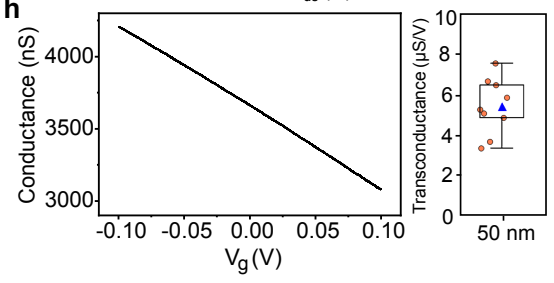

c

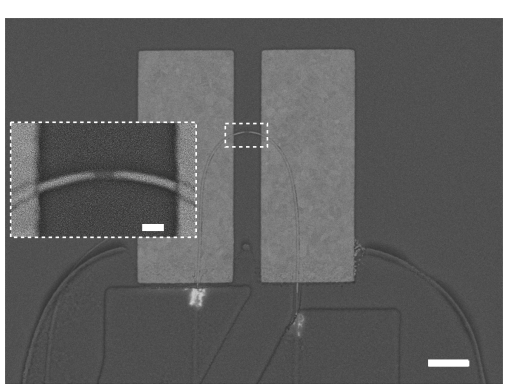

f 1000
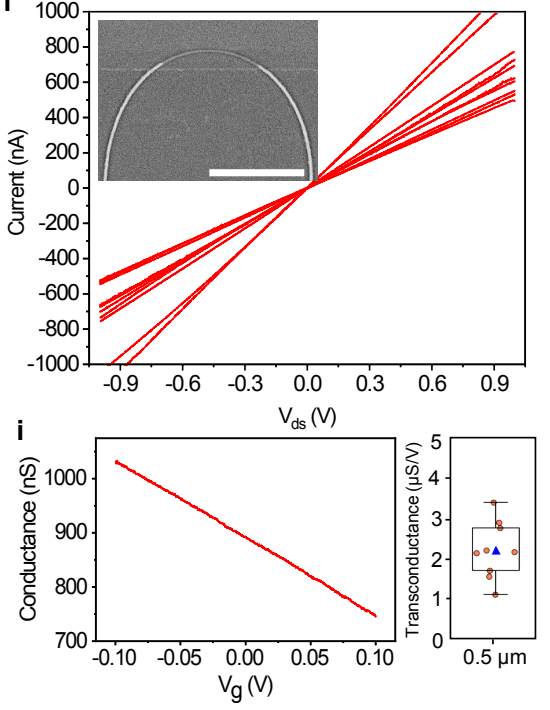

Bend-up

probe arm

d

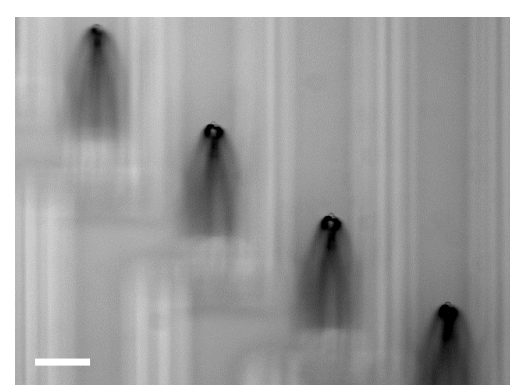

g
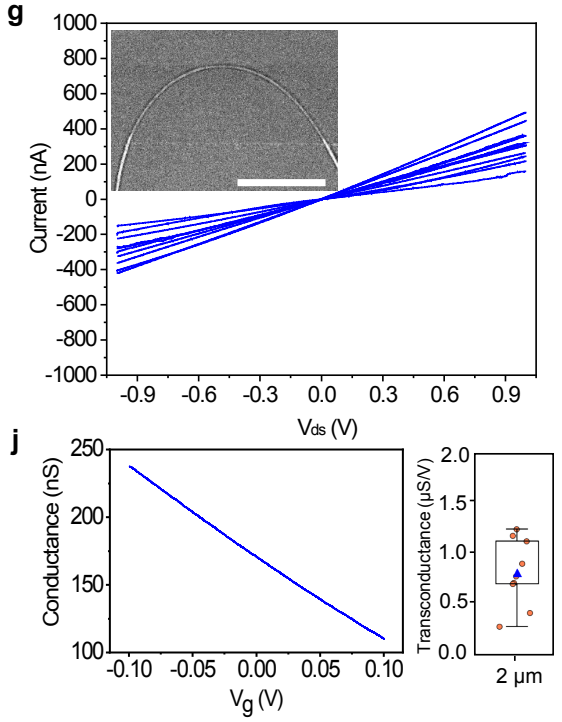


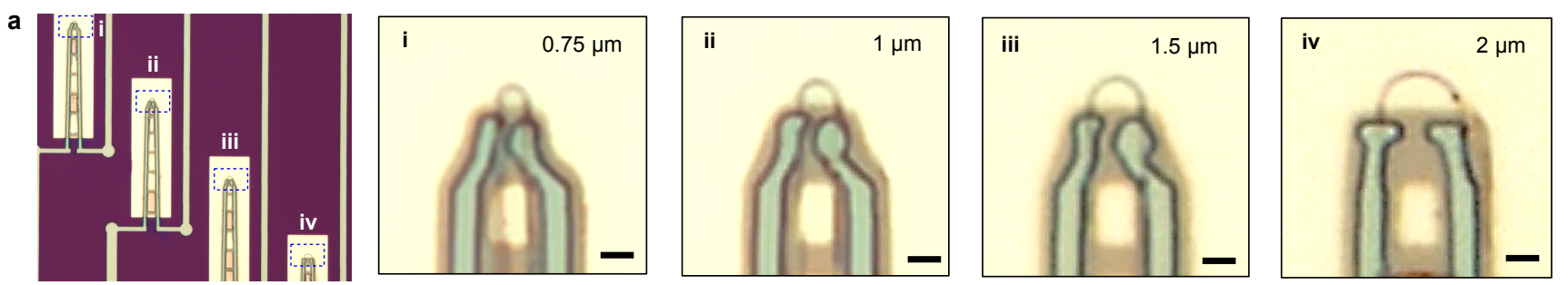

b
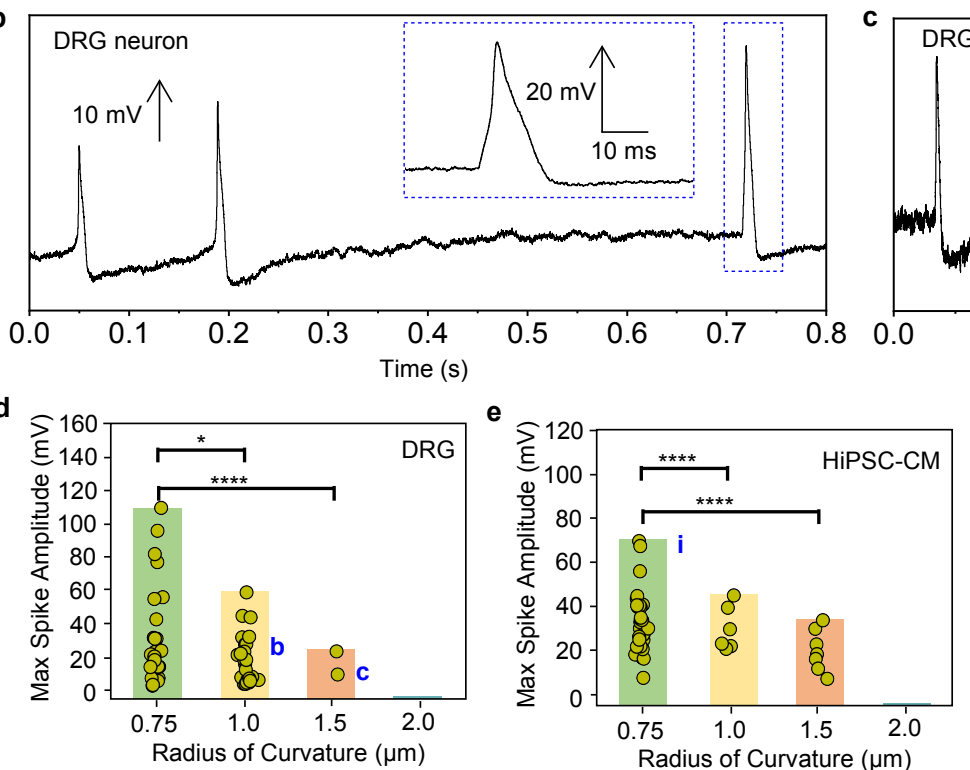

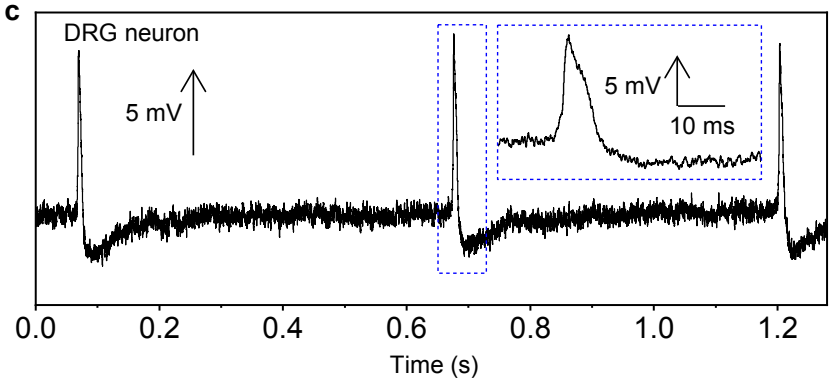

h

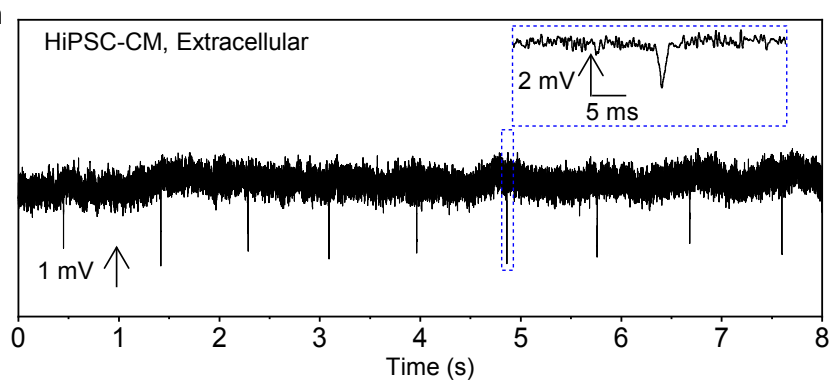

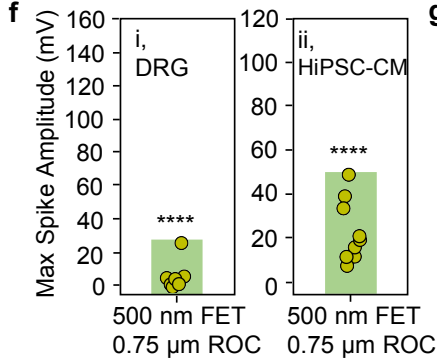
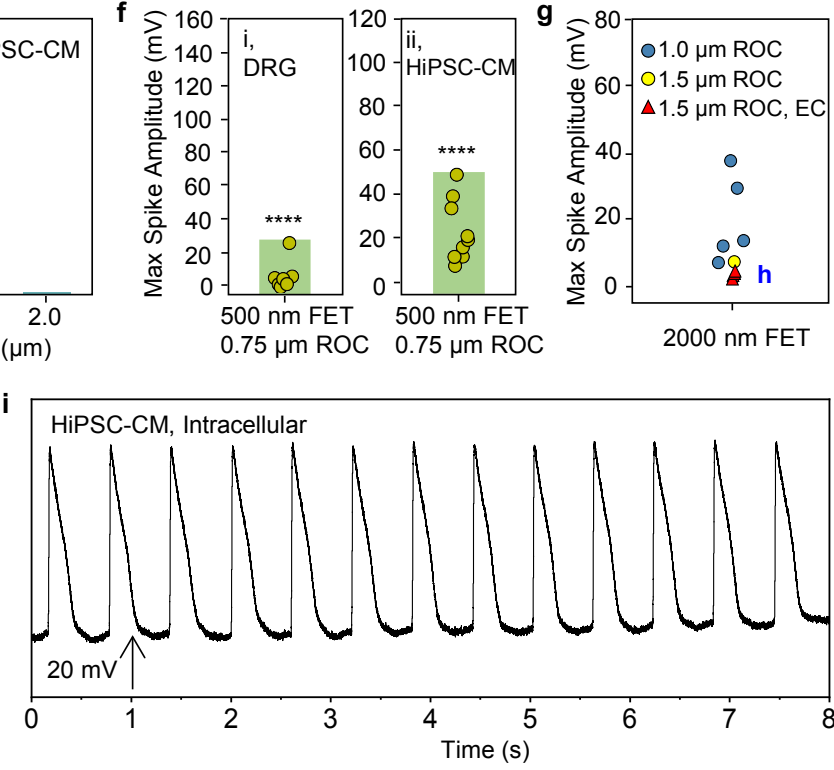
